\title{
Mapping flow distortion on oceanographic platforms using computational fluid dynamics
}

\author{
N. O'Sullivan, S. Landwehr, and B. Ward \\ School of Physics and Ryan Institute, National University of Ireland, Galway, Ireland \\ Correspondence to: B. Ward (bward@ nuigalway.ie) \\ Received: 18 October 2012 - Published in Ocean Sci. Discuss.: 9 November 2012 \\ Revised: 29 July 2013 - Accepted: 9 September 2013 - Published: 17 October 2013
}

\begin{abstract}
Wind speed measurements over the ocean on ships or buoys are affected by flow distortion from the platform and by the anemometer itself. This can lead to errors in direct measurements and the derived parametrisations. Here we computational fluid dynamics (CFD) to simulate the errors in wind speed measurements caused by flow distortion on the RV Celtic Explorer. Numerical measurements were obtained from the finite-volume CFD code OpenFOAM, which was used to simulate the velocity fields. This was done over a range of orientations in the test domain from -60 to $+60^{\circ}$ in increments of $10^{\circ}$. The simulation was also set up for a range of velocities, ranging from 5 to $25 \mathrm{~m} \mathrm{~s}^{-1}$ in increments of $0.5 \mathrm{~m} \mathrm{~s}^{-1}$. The numerical analysis showed close agreement to experimental measurements.
\end{abstract}

\section{Introduction}

Accurate measurements of the in situ wind speed are crucial for air-sea interaction studies. In most cases the wind speed is the primary driving force for surface mixing and air-sea exchange, and is therefore typically used as the main or even single parameter for the scaling of air-sea transfer rates. Fast measurements of the three components of the wind speed are used to directly measure air-sea fluxes with the eddy covariance method. However, flow distortion occurs when streamlines circumvent the research platform (see Fig. 1), and can lead to acceleration or deceleration and tilt of the wind vector.

One of the first attempts to define the error associated with flow distortion was a study conducted on the RV Aranda by Kahma and Lepparanta (1981). An empirical correction factor was developed from the in situ wind speed measure- ments taken on the ship's main tower and a bow mast, and by balloon tracking. Kahma and Lepparanta (1981) found that the distortion effect at the ship's main anemometer was on average $10 \%$ and very sensitive to the wind direction. The measurements taken with an anemometer mounted at a bow boom were however in agreement with free-stream measurements taken from balloonracking.

A series of complex potential flow models (i.e steady, inviscid, incompressible and irrotational flow) was used by Oost et al. (1994) to find corrections for flow distortion for the mean wind speed and the wind stress. For direct eddy covariance measurements, wind stress can be calculated from the covariance of the horizontal and vertical velocity fluctuations.

$$
\tau=\rho_{a} \cdot \overline{w^{\prime} u^{\prime}}
$$

Oost et al. (1994) found that the wind stress calculation can also be affected by the tilt of the mean flow away from the horizontal plane and estimated an additional factor of $6 \%$ per degree of tilt for this correction.. Oost et al. (1994) concluded that the numerical correction models were not accurate for the spatial definitions of the structure, and that wind tunnel testing of scaled physical models is more suitable.

Surry et al. (1989) and Thiebaux (1990) used wind tunnel testing of scaled physical models to estimate the air flow over the Canadian research ships RV Hudson and RV Dawson. The results showed an increase of $7 \%$ in air flow over anemometer sites located above the ship's bridge. Brut et al. (2002) used scaled physical models in a water flume, and showed that the mast on which the anemometer is mounted can have a dramatic effect on the airflow measurements.

An empirical estimate of the effect of flow distortion on flux estimates based on EC was derived by 


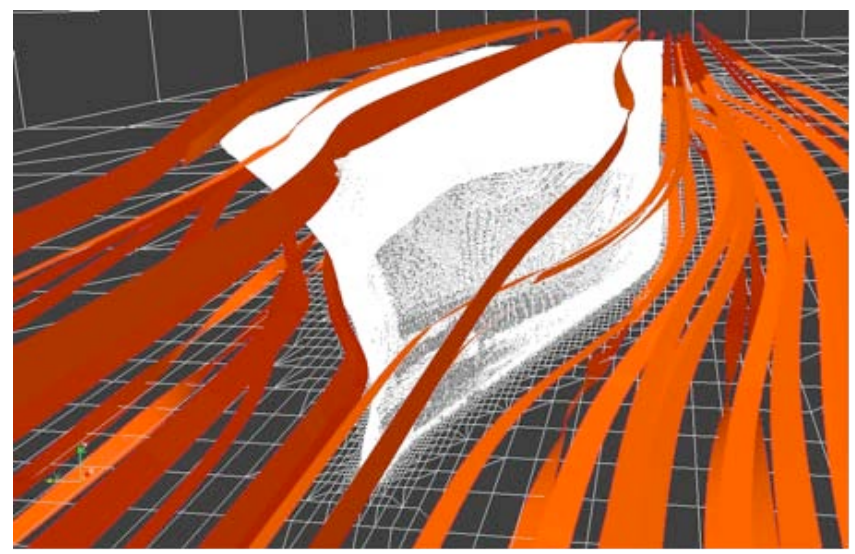

Fig. 1. Streamlines of distorted airflow by the research vessel's superstructure.

Edson et al. (1998) by comparing their own measurement on board the RV Wecoma to measurements taken on the research platform RP FLIP when both were within $50 \mathrm{~km}$ of each other. The measurements on RP FLIP were considered to be unaffected by flow distortion, because the anemometers were positioned on a long boom away from the superstructure. For relative wind directions between -120 and $+120^{\circ}$ on the bow of the RV Wecoma, Edson et al. (1998) found a $15 \%$ overestimation of the wind stress.

Errors in wind speed measurements caused by flow distortion are also problematic for the derivation of wind-speedbased parametrisations of the air-sea gas transfer velocity $k_{\mathrm{g}}$. These are usually of the form

$k_{\mathrm{g}}=A \cdot(u 10)^{B}$.

Griessbaum et al. (2010) used the updated GERRIS code (after Popinet, 2008) to simulate the effect of airflow distortion on wind speed measurements on the research vessels RV Hakuho Maru and RV Mirai. The estimated errors in the mean wind speed ranged from 4 to $+14 \%$. Depending on the exponent $B$ in Eq. (2), this can lead to possible biases in the gas transfer velocity $k_{\mathrm{g}}$ ranging from 30 to $50 \%$. Griessbaum et al. (2010) concluded that flow distortion effects could explain a part of the huge variance in gas transfer parametrisation: the bias in the transfer velocity varies with both wind speed and with relative wind direction. Therefore the bias in the gas transfer velocity $k_{\mathrm{g}}$ is unlikely to be the invariant from one cruise period to another, even if the same platform and anemometer setup is used.

Physical models have limitations but can be used to describe the mean flow distortion. However, in order to model the distortion effects on turbulent fluxes, the turbulent length scale must also be scaled (Popinet et al., 2004). The turbulent length scale describes the size of the large energycontaining eddies in a turbulent flow. To define these structures, a sub-scale large eddy simulation (LES) must be employed. The most appropriate available method to achieve this is to use computational fluid dynamics (CFD), which requires a numerical mesh that depends on the research platform's shape. The numerical mesh is used to solve partial differential equations between adjacent cells. CFD modelling for the quantification of flow distortion for wind speed measurements was first conducted by Yelland et al. (1998) for the RSS Discovery and the RSS Charles Darwin. They used the software package Vectis (a commercial software package which utilises a three-dimensional Reynolds-averaged Navier-Stokes (RANS) solver) to predict the airflow distortion at various anemometer sites. The simulations predicted that wind speed measurements are biased by approximately $10 \%$ at certain anemometer sites. These simulated predictions were used to correct inertial dissipation measurements of the drag coefficient for the four anemometers. This led to the difference in the average drag coefficients being reduced from a maximum of $20 \%$ for the uncorrected data to $5 \%$ or better for the corrected data (Yelland et al., 1998). Yelland et al. (2002) showed that the modelled flow distortion error agreed with the in situ experimental wind speed measurements to within $2 \%$ on various research ships and anemometer locations. These simulations also predicted the vertical displacement of the velocity in order to correct the inertial dissipation method for measuring fluxes. The simulation verification was provided by comparing inertial dissipation measurements of the wind stress, obtained from instrument sites that experienced a wide range of vertical displacements of air flow (Yelland et al., 2002). These showed a numerically modelled vertical displacement error ranging from 3.8 to $-15.2 \%$.

Popinet et al. (2004) used the more computationally expensive LES solver, which allowed for greater resolution in the turbulent regime of the simulation as well as more defined areas of recirculation on areas in the wake of the ship's superstructure. The Gerris open-source CFD solver was used, which solves three-dimensional, time-dependent Euler equations for an incompressible and inviscid fluid of constant density (Popinet, 2003). This paper involved both experimental and numerical data taken from the RV Tangaroa. Popinet et al. (2004) showed that the mean flow characteristics are only weakly dependent on the ship motion, ship speed, wind speed or sea state, but strongly dependent on the relative wind direction. For well-exposed anemometers, the wind speed measurements had a $5 \%$ error, and for anemometers in the wake of the ship's superstructure, there were normalised standard deviations of up to $40 \%$.

CFD has become an established method for correcting the errors associated with direct flux measurements, and this paper deals with a CFD flow distortion study for the RV Celtic Explorer. In this paper we report firstly on a computer-aided design (CAD) model of a sonic anemometer mounted on a mast, which represents the physical measurements on the RV Celtic Explorer. Then the in situ measurements are compared to the CFD solution, and from this a correction is developed for the RV Celtic Explorer. We then use the CFD results to 

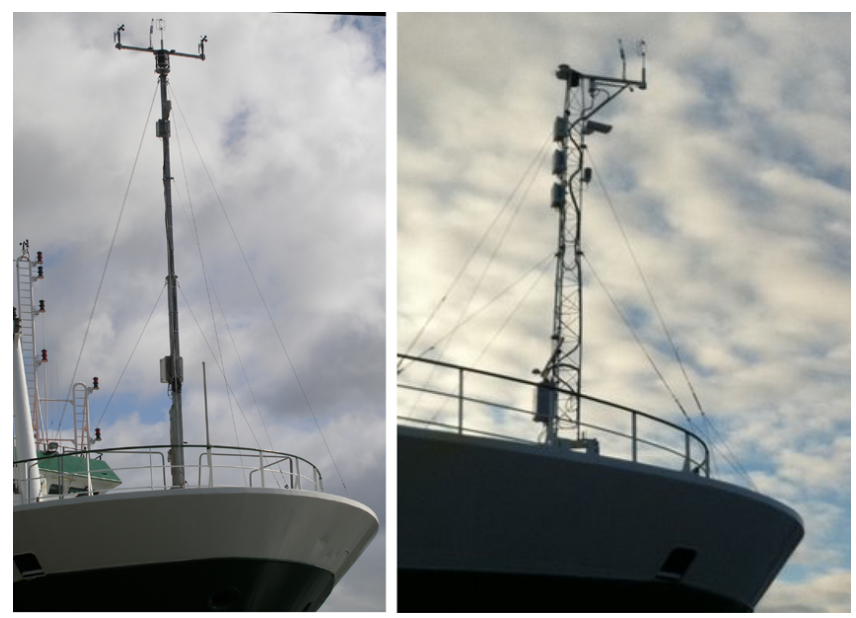

Fig. 2. Photos of the two masts that have been used in this study: left is the single pole mast with a crossbeam for the anemometers. Right shows the triangular lattice mast with the sonic anemometer located on a boom towards the bow.

determine the ideal location for wind speed measurements on the vessel. Furthermore, we investigate the effect of varying pitch angles on the measurement data. Finally, we test two mast designs with three instrumentation setups for the optimum experimental design. Our conclusions are provided in the final section.

\section{In situ measurements}

Measurements were conducted on the RV Celtic Explorer, where, during the period of this project, two masts were used (Fig. 2). The objective of the project was to develop an eddy correlation air-sea flux system for the research vessel. Micro meteorological instrumentation consisted of a Gill R3A sonic anemometer with a Crossbow NAV440 inertial motion unit as well as a Licor LI-7500 $\mathrm{CO}_{2} / \mathrm{H}_{2} \mathrm{O}$ analyser. The mean meteorological measurements were performed using a Young anemometer (wind speed and direction), a Vaisala temperature and humidity probe, a Druck atmospheric pressure sensor, an Eppley radiometer (infrared radiation) and an Eppley pyranometer (shortwave radiation). The micrometeorological and mean data were logged on a Moxa UC-7410 embedded computer and a Campbell CR3000 data logger at sample rates of 10 and $0.1 \mathrm{~Hz}$, respectively. These in situ data provided an opportunity to compare the CFD-modelled data described below.

\section{CFD modelling}

The finite-volume CFD code OpenFOAM 2.0.1 (OpenCFD, 2012) was used to simulate the velocity fields around the RV Celtic Explorer. OpenFOAM is a C++ library used primarily to create executables, known as applications. The appli- cations fall into two categories: solvers, designed to solve a specific problem in continuum mechanics; and utilities, designed to perform tasks that involve data manipulation. Two standard solvers were used for the simulations presented here:

- PotentialFOAM - a potential flow solver which can be used to generate starting fields for full Navier-Stokes codes and reduces the normal run up time instabilities associated with steady-state simulations (OpenFOAM, 2011).

- SimpleFOAM - this uses the semi-implicit method for pressure-linked equations (SIMPLE) algorithm, which allows for coupling of the Navier-Stokes equations with an iterative procedure (openfoamwiki, 2010).

The simulation properties follows the prescribed method of setup conducted by Gagnon and Richard (2010) for the OpenFOAM implementation of the simpleFOAM steadystate algorithm. They were tested against the Ahmed body (a simplified, standardised car body used in CFD testing) wind tunnel data. There are an extensive number of experimental wind tunnel datasets, which are widely used in the validation of external aerodynamics simulations in CFD. Testing against these experimental data is considered an acceptable comparison, as the Ahmed body creates defined vortices in its wake region. The Ahmed body model also has an angled front section which allows for a defined comparison of lift and drag coefficients. The conclusion from Gagnon and Richard (2010) showed that the chosen simulation's setup using the simpleFOAM algorithm had a $10 \%$ difference from experimental wind tunnel testing data. A time-varying velocity was applied from 5 to $25 \mathrm{~m} \mathrm{~s}^{-1}$ in steps of $0.5 \mathrm{~m} \mathrm{~s}^{-1}$ at the inlet boundary condition, which is a spatial specification of values at the domain inlet. The temporal discretion scheme (computational time step equations control) between points was defined to allow each velocity input to pass through the domain 10 times (OpenFOAM, 2011).

A Courant number of less than 1 was used for temporal accuracy and numerical stability (Souza, 2005). The Courant number is the input speed multiplied by the ratio of the time step length to the cell length. This ratio is the time required for a quantity or fluid particle to be convected over a small distance. This was also applied to improve the computational cost on running a range of velocity inputs, as well as to obtain a greater array of values to compare with experimental data. The influence of boundaries is minimised by using a ratio between model and domain size of less than $1 \%$. The walls which are the left, right and top boundaries of the simulation domain have little effect on the model, and therefore these boundary conditions were set up as symmetry planes. A symmetry plane is where the normal velocity is zero and the normal gradients of all other variables are also zero (Souza, 2005). The highly effected regions of the simulations and 
thus the most significant boundary conditions are the vessel surface and the floor surface (the lower boundary of the simulation domain). In accordance with Gagnon and Richard (2010), a classical log-law wall function was applied to the domain floor and vessel's surface for the turbulence characteristics of $k$ (specific kinematic viscosity) and $\Omega$ (specific dissipation rate). The $k-\Omega$ shear stress transport (SST) turbulence model (Menter, 1993) was used for a turbulent intensity of $4 \%$ for all boundary conditions.

A Reynolds number of $10^{8}$ referenced from Popinet et al. (2004) for airflow at the air-sea boundary layer in the ocean was used to calculate the kinematic viscosity of the fluid. This model is a two-equation eddy viscosity model, and is usable all the way down to the wall through the viscous sub layer. The model was used for its proven reliability in separation zones as well as its ability to blend a good free-stream model with a good boundary layer model (Gagnon and Richard, 2010). The outputted calculations from the simulations contain logs for $U$ (velocity), $P$ (pressure) and $k$.

The CAD models were generated using the 3-D solid modelling software Blender 2.60 (Blender, 2011). Figure 3 shows the generated three-dimensional model and a cross section of the mesh refinement containing hexahedron (hex) and split-hexahedron (split-hex) cells around the RV Celtic Explorer CAD model. Also shown are the four anemometer measurement positions - the bow-mast Gill sonic (BMS), Young's mean 1 (YM1), Young's mean 2 (YM2) and the ship bridge deck (SBD). The CAD model was scaled to $1: 10$, which in turn defined a simulation domain size of $37.85 \mathrm{~m} \times 20.2 \mathrm{~m} \times 20.2 \mathrm{~m}$ for flows directly over the bow. This CAD model scale and model domain size was chosen to give a $1 \%$ blockage area (area ratio between the inlet of the simulation domain and the CAD model) of the inlet section of the wind tunnel by the test geometry as described by Castro and Robins (1977). This allows for the flow to completely stabilise within the simulation domain. In the case of the RV Celtic Explorer, the size of the domain is also dependent upon the ship's orientation to the flow. The width of the domain can therefore increase to over $40 \mathrm{~m}$ for flows over the ship's beam. CAD models scaled to $1: 1$ of the two masts (Fig. 2) were also created and implemented with the same simulation setup (i.e OpenFOAM numerical solvers, boundary conditions and turbulence model) as the RV Celtic Explorer simulations. A $1 \%$ inlet blockage area was also applied to the domain size and model scale. The mast CAD models were contained within a simulation domain of $46.9 \mathrm{~m} \times 24.9 \mathrm{~m} \times 24.9 \mathrm{~m}$. The mast CAD models were also configured with three different instrumentation setups, containing different sonic anemometer and Licor gas analyser setups.

Finally, a simulation was implemented where the effect of the pitch angle of the ship on the wind speed determination was evaluated. The CAD model was tilted within the domain through orientations ranging from 0 to $6^{\circ}$ in steps of $2^{\circ}$. The

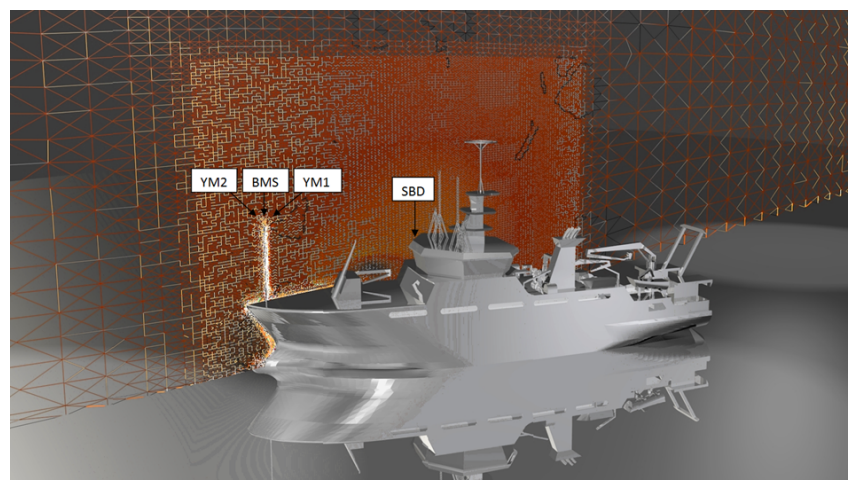

Fig. 3. RV Celtic Explorer model with cross section of hex mesh and relative position of experimental anemometers - bow-mast Gill sonic (BMS), Young's mean 1 (YM1), Young's mean 2 (YM2) and ship bridge deck (SBD).

domain size and simulation setup remained the same for this model as previously used for the RV Celtic Explorer simulations.

The CAD models were imported into OpenFOAM as an interchangeable 3-D file format. In the vessel simulations this was done over a range of orientations in the test domain, from -60 to $+60^{\circ}$ in increments of $10^{\circ}$, giving 13 model orientations within the simulation domain. The simulation was set up for 41 different velocities, ranging from 5 to $25 \mathrm{~m} \mathrm{~s}^{-1}$ in increments of $0.5 \mathrm{~m} \mathrm{~s}^{-1}$.

The vertical orientation of the model was carried out over a range of orientations in the test domain, from 0 to $+6^{\circ}$ in increments of $2^{\circ}$, giving four model orientations within the simulation domain. The simulation was also setup for 41 different velocities, ranging from $5 \mathrm{~m} \mathrm{~s}^{-1}$ to $25 \mathrm{~m} \mathrm{~s}^{-1}$ in increments of $0.5 \mathrm{~m} \mathrm{~s}^{-1}$.

The meteorological mast setup comparisons were performed for $0-60^{\circ}$. This simulation setup was also done for 41 different velocities, ranging from 5 to $25 \mathrm{~m} \mathrm{~s}^{-1}$ in increments of $0.5 \mathrm{~m} \mathrm{~s}^{-1}$. The meteorological mast setup comparisons were performed for 2 different mast designs (Mast1 telegraph pole design and Mast2 triangular lattice design) and 3 different instrumentation setups (Gill sonic anemometer, Gill sonic anemometer/Licor gas analyser and Campbell sonic anemometer/Licor gas analyser), giving 42 simulations in total. The overall total number of simulations run from all the variations tested was 59 .

The OpenFOAM code was compiled with gcc-4.4 and Open MPI, and ran on 2 cluster computers: a Bull NovaScale R422-E2 cluster with 64 compute nodes and an SGI Altix ICE 8200EX cluster with 320 compute nodes. For the former, each computer node has two $2.8 \mathrm{GHz}$ Intel (Nehalem EP) Xeon X5560 quad-core processors and 48 GB of RAM, resulting in a total of 512 cores and 3072 GB of RAM available for jobs. For the latter, each compute node has two Intel (Westmere) Xeon E5650 hex-core processors and 24 GB of RAM, with a total of 3840 cores and $7680 \mathrm{~GB}$ of RAM 
(a)
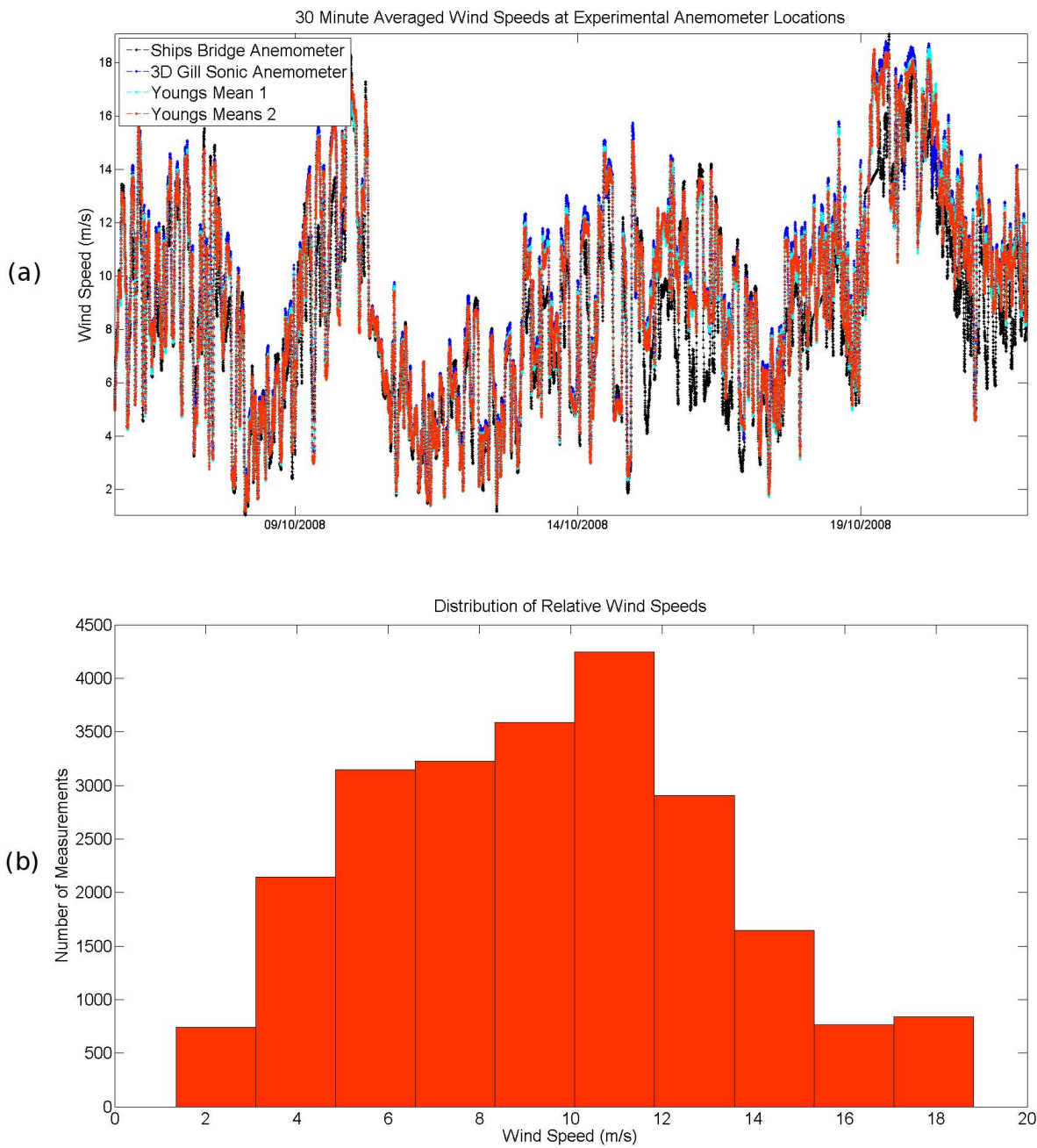

Fig. 4. (a) Thirty-minute-averaged true-wind-corrected measurements for the BMS, YM1, YM2 and SBD anemometers. (b) Histogram of distribution of measured wind speeds for chosen cruise period from the BMS anemometer.

available for jobs. There was a total run time of 14.5 days for the completed code using both computers. Each case was run on 64 parallel processors per case.

\section{Results and discussion}

In accordance with Popinet et al. (2004), two initial assumptions were made:

1. The wind speeds measured at different locations should scale linearly with some reference velocity, meaning the fluid flow is essentially independent of the Reynolds number.

2. The averaged velocity depends only on the relative wind direction.

The first assumption is justified as the Reynolds number ( $\sim 10^{8}$ based on ship length and $10 \mathrm{~m} \mathrm{~s}^{-1}$ wind speed), and is well within the asymptotic regime for flow around a solid obstacle (Popinet et al., 2004). We investigate the linearity of the scale of error using highly resolved wind speed runs (see Sect. 4.1).

The second assumption chooses to neglect the influence of the sea conditions as well as ship motion. It is extremely difficult to simulate ship movement (yaw, pitch and roll), which leads to large computational costs. We do, however, investigate flow for various fixed pitch angles (see Sect. 4.3).

The experimental results were taken during a RV Celtic Explorer cruise in October 2008 using the Mast1 design instrumentation setup (Fig. 2). The results are processed to give a true-wind velocity value corrected for the ship with reference to the Earth's surface and true north. This is done for YM1 and 2 anemometers and the BMS anemometer on the bow mast as well as the SBD anemometer. A $30 \mathrm{~min}$ averaged time series of data for the chosen cruise period is shown in Fig. 4a. This gives a mean difference from the 
(a)

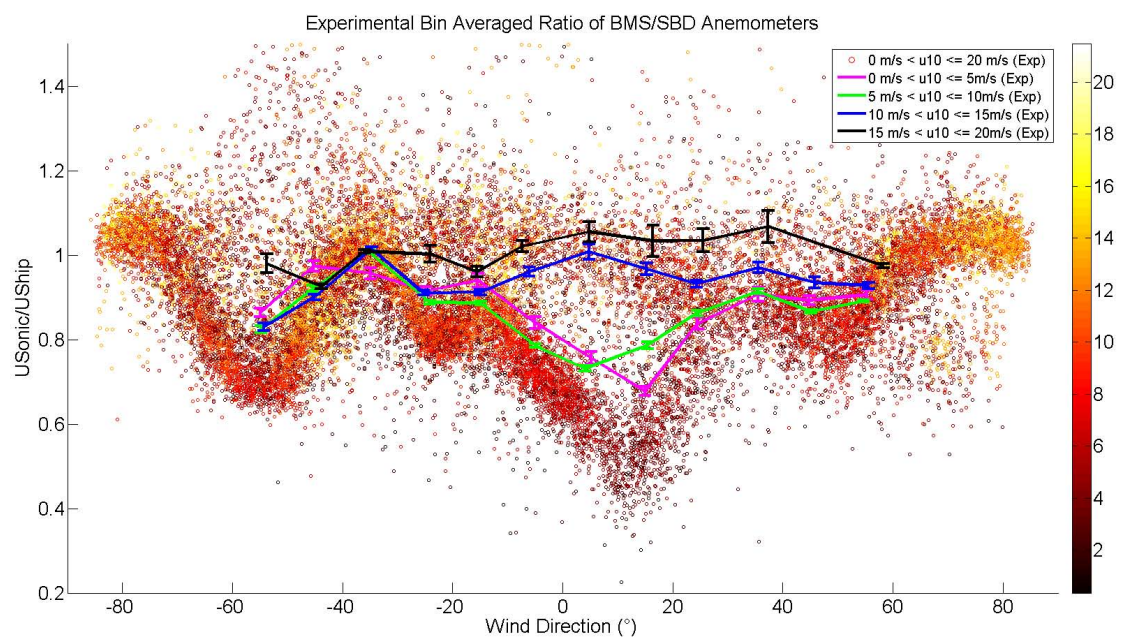

(b)

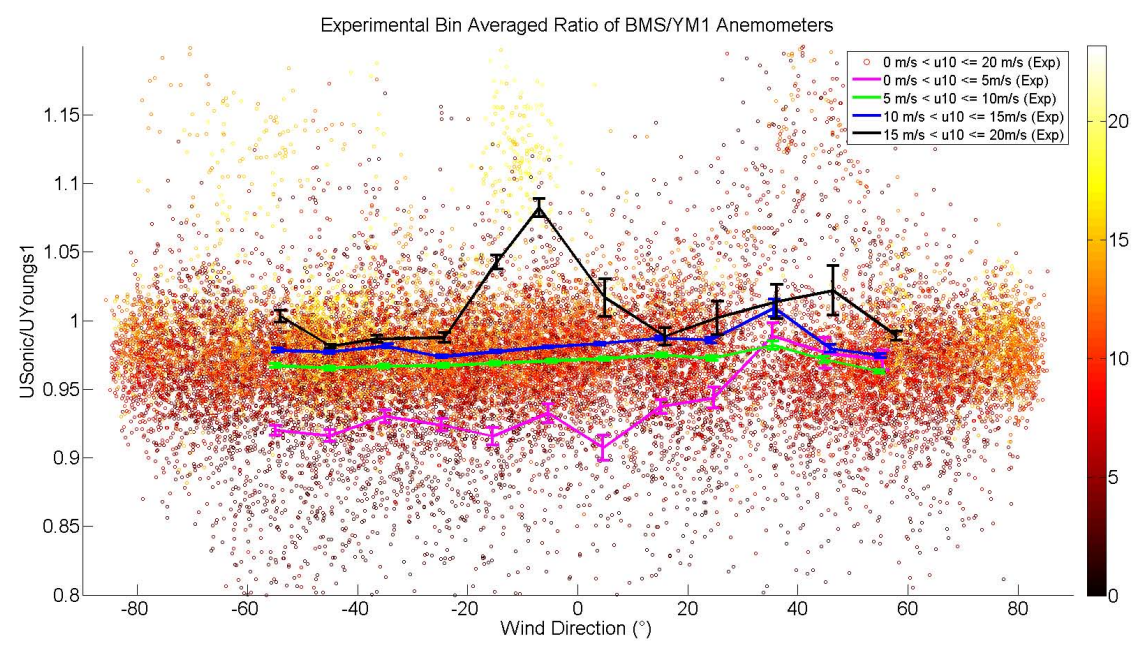

Fig. 5. (a) Experimental bin-averaged wind speed line plots between the BMS and the SBD anemometers over a range of direction from -60 to $+60^{\circ}$, also with experimental scatter plot of full wind speed range and standard deviation error bars. (b) Experimental bin-averaged wind speed line plots between the BMS and the YM1 anemometers over a range of direction from -60 to $+60^{\circ}$, also with experimental scatter plot of full wind speed range and standard deviation error bars.

BMS anemometer location to the SBD anemometer location of $1.28 \mathrm{~m} \mathrm{~s}^{-1}$. From the BMS anemometer to the YM1 anemometer a mean difference of $0.27 \mathrm{~m} \mathrm{~s}^{-1}$ is recorded. In Fig. $4 \mathrm{~b}$, the distribution of velocities showed a lack of data at wind velocities greater than $15 \mathrm{~m} \mathrm{~s}^{-1}$.

The scatter plot Fig. 5a shows the ratio of experimental $1 \mathrm{~min}$ averaged wind speeds from the BMS and the SBD anemometer with respect to the wind direction. Also shown are the bin-averaged data for different wind speed ranges. Figure 5a shows how the change in wind speed affects the experimental ratio in measurements between the two anemometers, and that increased distortion effects occur at 10 and $-60^{\circ}$ to the bow. This gives a representation of the wind speed dependence (i.e the deviation of flow distortion error scaling from linearity as the measured wind speed increases), and also shows that the highest level of the flow distortion ranges from -5 to $+30 \%$. As the orientation tends towards the negative direction, a higher distortion error can be seen because of the recirculation caused by a crane which was mounted on the port-side of the vessel. However, this is not the case in the positive wind direction, as is evident in Fig. 5a.

Figure $5 b$ shows the ratio of experimental 1 min averaged wind speeds from the BMS anemometer and the YM1 starboard anemometer (refer to Fig. 3). Here the ratio decreases as a function of wind speed (i.e. the ratio tends towards 1 as the wind speed increases). From this plot, the magnitude of the flow distortion error range was 5 to $10 \%$.

\subsection{Vessel simulations}

The vessel simulations were carried out over a range of orientations from -60 to $+60^{\circ}$ in increments of $10^{\circ}$. The simulation was also set up for a range of velocities, ranging from 5 


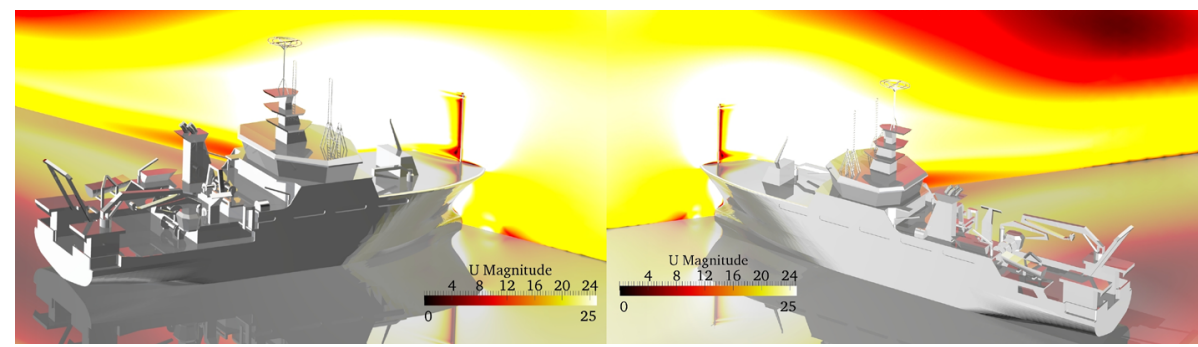

Fig. 6. (a) Flow speed over the ship for -60 and $+60^{\circ}$ to the bow for a steady-state input stream of $25 \mathrm{~m} \mathrm{~s}^{-1}$.

Table 1. Mean differences between numerical and experimental anemometer ratio bin-averaged data in Fig. 8 .

\begin{tabular}{lcccc}
\hline $\begin{array}{l}\text { Anemometer Ratio } \\
\text { Differences }\end{array}$ & $0-5 \mathrm{~m} \mathrm{~s}^{-1}$ & $5-10 \mathrm{~m} \mathrm{~s}^{-1}$ & $10-15 \mathrm{~m} \mathrm{~s}^{-1}$ & $15-20 \mathrm{~m} \mathrm{~s}^{-1}$ \\
\hline BMS/SBD & $3.78 \%$ & $2.71 \%$ & $6.61 \%$ & $0.50 \%$ \\
BMS/YM1 & $6.25 \%$ & $3.12 \%$ & $2.60 \%$ & $2.88 \%$ \\
BMS/YM2 & $6.53 \%$ & $3.62 \%$ & $3.93 \%$ & $3.92 \%$ \\
\hline
\end{tabular}

to $25 \mathrm{~m} \mathrm{~s}^{-1}$ in increments of $0.5 \mathrm{~m} \mathrm{~s}^{-1}$. Cross-sectional contour plots for -60 and $+60^{\circ}$ inflow to the bow are shown in Fig. 6. These plots show the change in the velocity profile for the associated change in direction produce different wake structures in front of the bow. It can be seen for $-60^{\circ}$ (Fig. 6a) that there is a higher gradient of turbulence in the wake of the bow mast. This is also apparent in the experimental results shown in Fig. 5a. This is due primarily to the presence of a bow-deck crane on the port side causing increased distortion effects. This verifies the validity of the simulation results as spatially accurate to the physical reality of the experimental results.

A free-stream undistorted measurement was unavailable from the current dataset. In previous works (e.g. Popinet et al., 2004), a reference measurement location was taken as the free-stream undistorted flow measurement (measurements of wind speed external to a research vessel are almost impossible to attain). Thus, in order to validate the simulations directly from the experimental measurements, a ratio method between chosen anemometers was conducted following the approach of Popinet et al. (2004). The comparison of the ratio between simulated wind speeds from a referenced location and another location in the numerical domain should predict the ratio in experimental measurements over this same space, thus giving an estimate of the accuracy of the simulations to predict a free-stream velocity occurring away from the vessels superstructure. This method is valid due to the fact that relative position of the anemometers does not change in time or space. The ratio across the prescribed anemometer locations was calculated in the simulation results. Figure 7 shows CFD velocities of flow onto the bow ranging from -60 to $+60^{\circ}$.

Figure $7 \mathrm{a}$ and $\mathrm{b}$ respectively show the ratio between the BMS anemometer and the YM1/2 starboard/port anemome- ters. The plots show peaks in the simulated error as the inflow direction aligns with the vertical (T-bar) section of the mast (see Fig. 3), thus causing elevated distortion since the instrument measurement location is in its wake. This is also apparent in Fig. 5b, where a similar range of errors $(-1$ to $10 \%$ ) is present, and also contains the same peak in the negative direction. In Fig. 7c the predicted errors between the BMS anemometer and the SBD anemometer show elevated distortion in the negative direction as a result of the bowdeck crane. Comparing Fig. 7c to Fig. 5a, the same range of errors can be seen. In Fig. 7d we see the surface plot prediction of errors for the BMS anemometer location, with respect to the difference from the free-stream velocity, which was taken from the model output for a position $20.5 \mathrm{~m}$ upstream of the bow (this is the distance between the BMS and SBD anemometers in Fig. 3).

Figure $8(\mathrm{a}, \mathrm{b}, \mathrm{c})$ shows the ratio between the various anemometers for both the experimental and modelled data, where the modelled data were derived from the correction factor, shown in Fig. 7 (a, b, c). The differences between the numerical and experimental bin-averaged data are presented in Table 1. The differences between the numerical and experimental ratios for the BMS / YM1 and BMS / YM2 are consistent with each other, with the percentage difference decreasing for increasing wind speeds. However the difference between the BMS / SBD ratio is not consistent with this, and there is a relatively large error for the $10-15 \mathrm{~m} \mathrm{~s}^{-1}$ range. This is not altogether surprising as there is a $20.5 \mathrm{~m}$ distance between the SBD and BMS anemometers (compared to $0.5 \mathrm{~m}$ between the BMS and YM1/YM2 anemometers). It should be noted the numerical model prediction shows the same trend in wind speed dependence shown in the experimental measurements bins (i.e the deviation of flow distortion error scaling from linearity as the numerical wind speed 


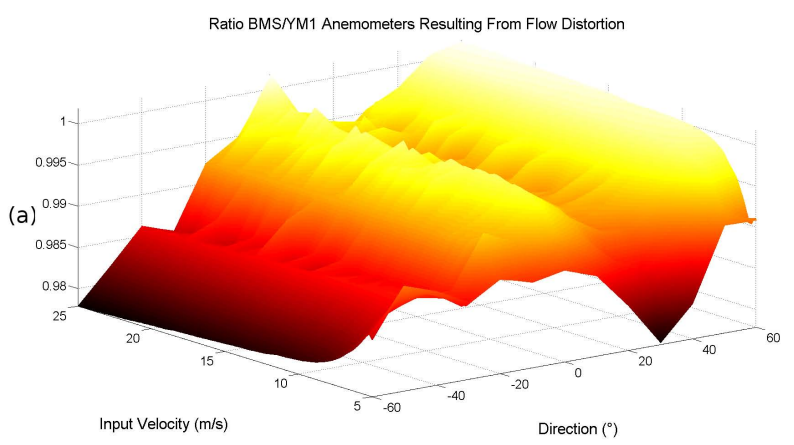

Ratio BMS/M2 Anemometers Resulting From Flow Distortion

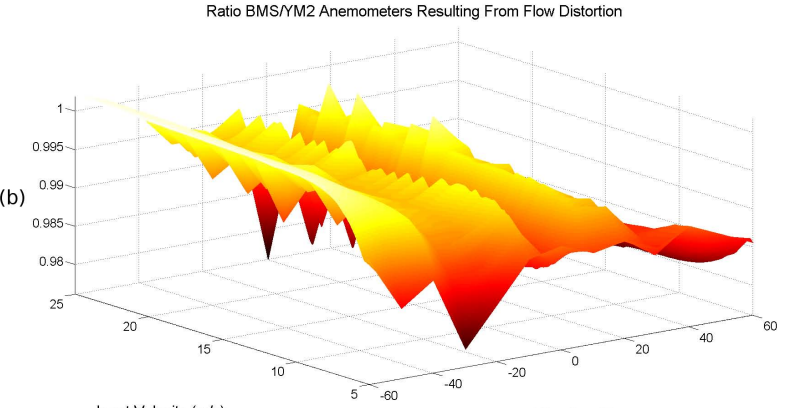

Input Velocity (m/s)

Direction $\left(^{\circ}\right)$

Ratio BMS/SBD Anemometers Resulting From Flow Distortion

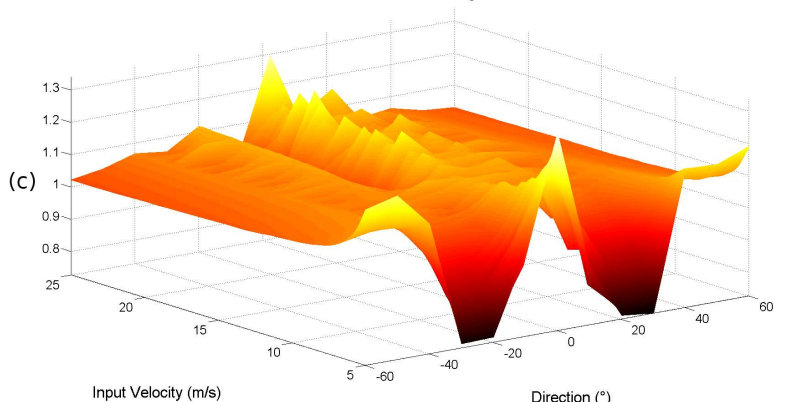

Ratio BMS/Free-stream Resulting From Flow Distortion

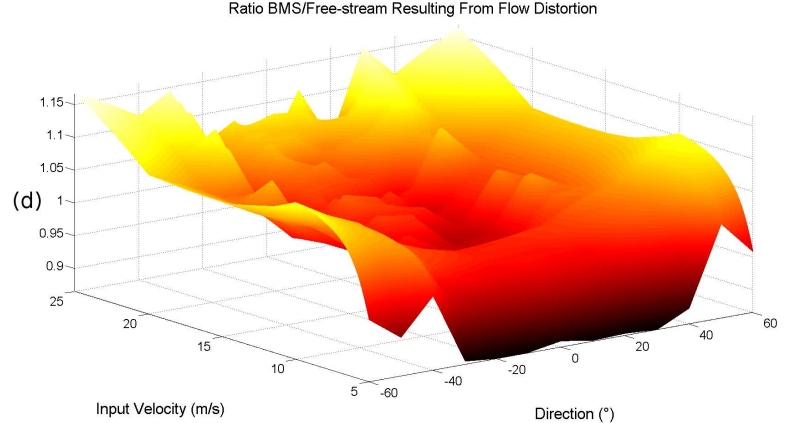

Fig. 7. Surface plot corrections for ratios between (a) BMS and YM1 starboard, (b) BMS and SBD, (c) BMS and YM2 port anemometer (d) BMS and free-stream velocity.

scales upwards). Figure $7 d$ corresponds to Fig. $8 d$, where the error found from the evaluated surface plot to free stream was extracted from the measured BMS anemometer. This gives a corrected plot of the data (in black) for the duration of the chosen cruise period. The plots show a correction range from 2.4 to $-1.0 \mathrm{~m} \mathrm{~s}^{-1}$, with a root-mean-square value of $0.43 \mathrm{~m} \mathrm{~s}^{-1}$. (a)

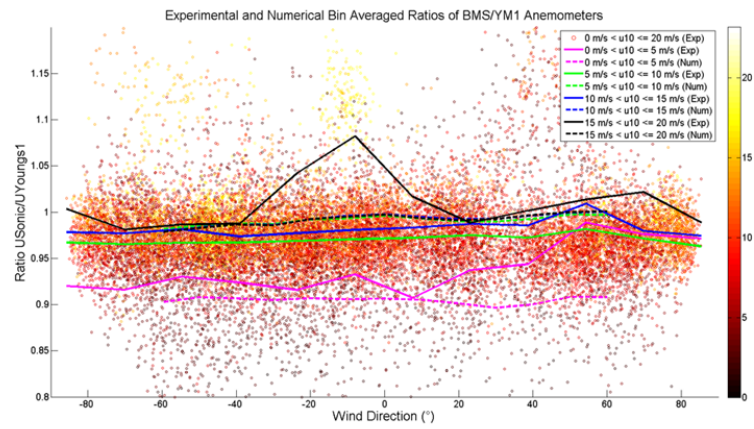

(b)

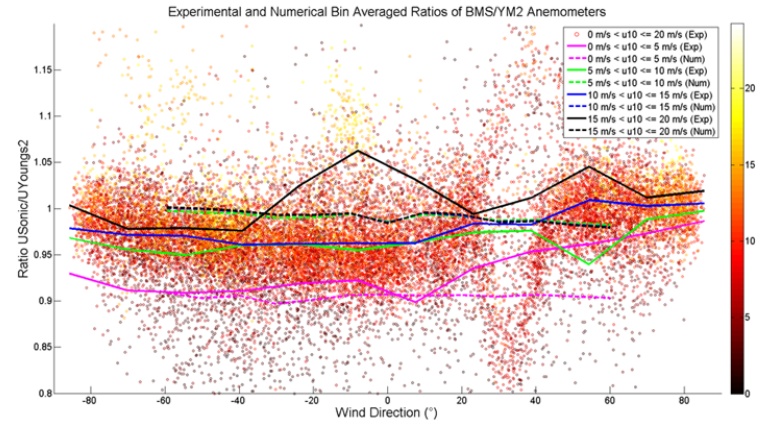

(c)
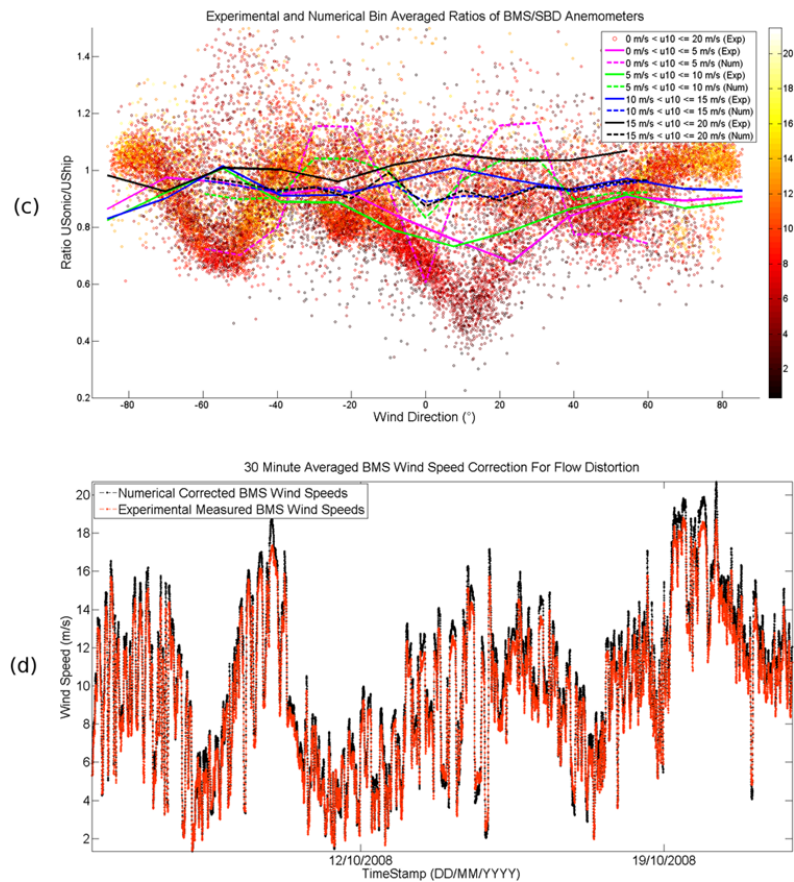

Fig. 8. Bin averages of the measured and modelled wind speed ratios as a function of the relative wind direction for (a) BMS and YM1 anemometers (b) BMS and YM2 anemometers (c) BMS and SBD anemometers individual measurements are shown as scatter plot with the wind speed as color code. (d) Time series of BMS and free-stream velocity. 


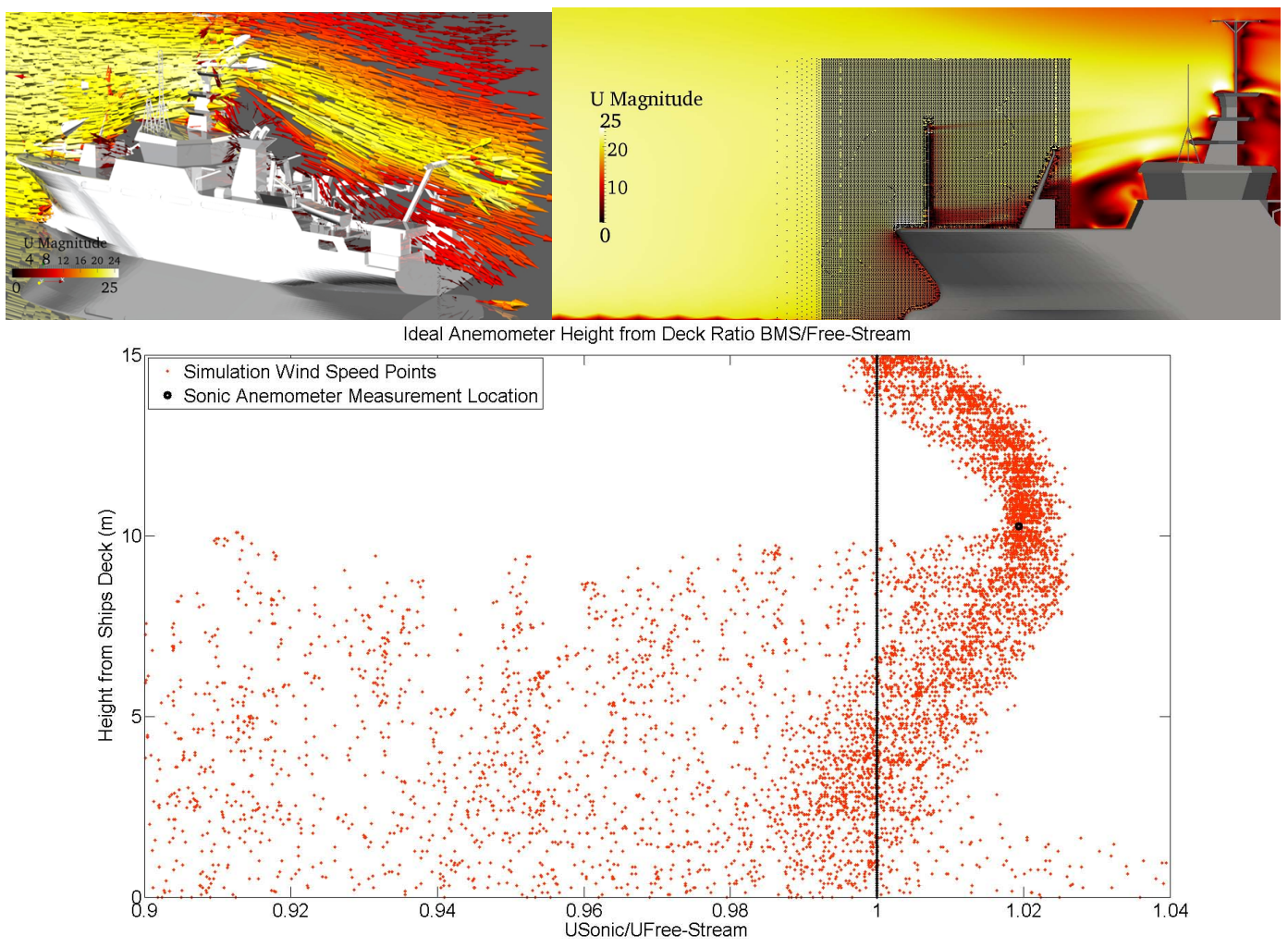

Fig. 9. Top left: cross-sectional velocity vector plot from 5 to $25 \mathrm{~m} \mathrm{~s}^{-1}$. Top right: grid location for determination of optimised position of the BMS. Bottom: ratio of BMS anemometer to free stream for the data points in the grid above. The black dot indicates the current location of the BMS anemometer.

\subsection{Ideal anemometer locations}

A vector plot of the velocity profile at $0^{\circ}$ inflow to the bow is shown in Fig. 9a. This plot was used to define the areas of highly deviated airflow, showing high distortion in the wake of any deck-mounted structure. Deviations from the mean flow and recirculation areas can be seen upwind of the bridge deck. Highly deviated areas with recirculation can also be seen in the wake of the bridge deck. This describes the optimum area for positioning of anemometers, which should be as far towards the bow as possible, and facing into the mean free-stream airflow. In Fig. 9b, there is a high-resolution grid (20 955 points) within this defined area, where velocity calculations were recorded for each point. The ratio to freestream velocity for the chosen points ahead of the mast position were plotted for change in height (Fig. 9c). This shows that the BMS anemometers current position is within a wake region caused by a forward velocity gradient from the bow; therefore the height should ideally be increased to $>14 \mathrm{~m}$. For the conditions of this numerical experiment (i.e. $0^{\circ}$ wind direction at $25 \mathrm{~m} \mathrm{~s}^{-1}$ ), the least distorted location for the anemometer was found to be at the bow, close to the deck of the ship. However, this location would likely be different for different flow conditions. The most accurate velocity position outside of this acceleration zone shows that the BMS anemometer should be placed $3.2 \mathrm{~m}$ ahead of the bow and higher than $14 \mathrm{~m}$ above the ship's deck.

\subsection{Vertical orientation}

Numerical experiments were conducted for pitch angles from 0 to $6^{\circ}$ in increments of $2^{\circ}$ for velocity ranges of 5 to $25 \mathrm{~m} \mathrm{~s}^{-1}$. Figure $10 \mathrm{a}$ shows high-resolution velocity plots for a wind input of $25 \mathrm{~m} \mathrm{~s}^{-1}$ and for a model tilt angle of $0^{\circ}$ and $6^{\circ}$ along its $y$ axis. What is most striking is the change in the velocity profile across the length of the ship. Focussing on the BMS probe location (Fig. 10b), the effect of changing the ship's pitch angle is shown. The vertical component of the wind is most sensitive to the change in pitch angle (not shown). The vertical orientation of the ship effectively creates a larger area of blockage with increasing pitch angle, resulting in a maximum distortion effect of $2.5 \%$ at a high wind speed of $20 \mathrm{~m} \mathrm{~s}^{-1}$.

\subsection{Meteorological mast comparison}

The meteorological mast setup simulations were carried out for two individual mast designs (see Fig. 2) with different instrumentation attached, which involves a Gill R3A sonic anemometer, a Campbell sonic anemometer and a Licor LI7500 gas analyser. Both mast designs and the instrumentation 


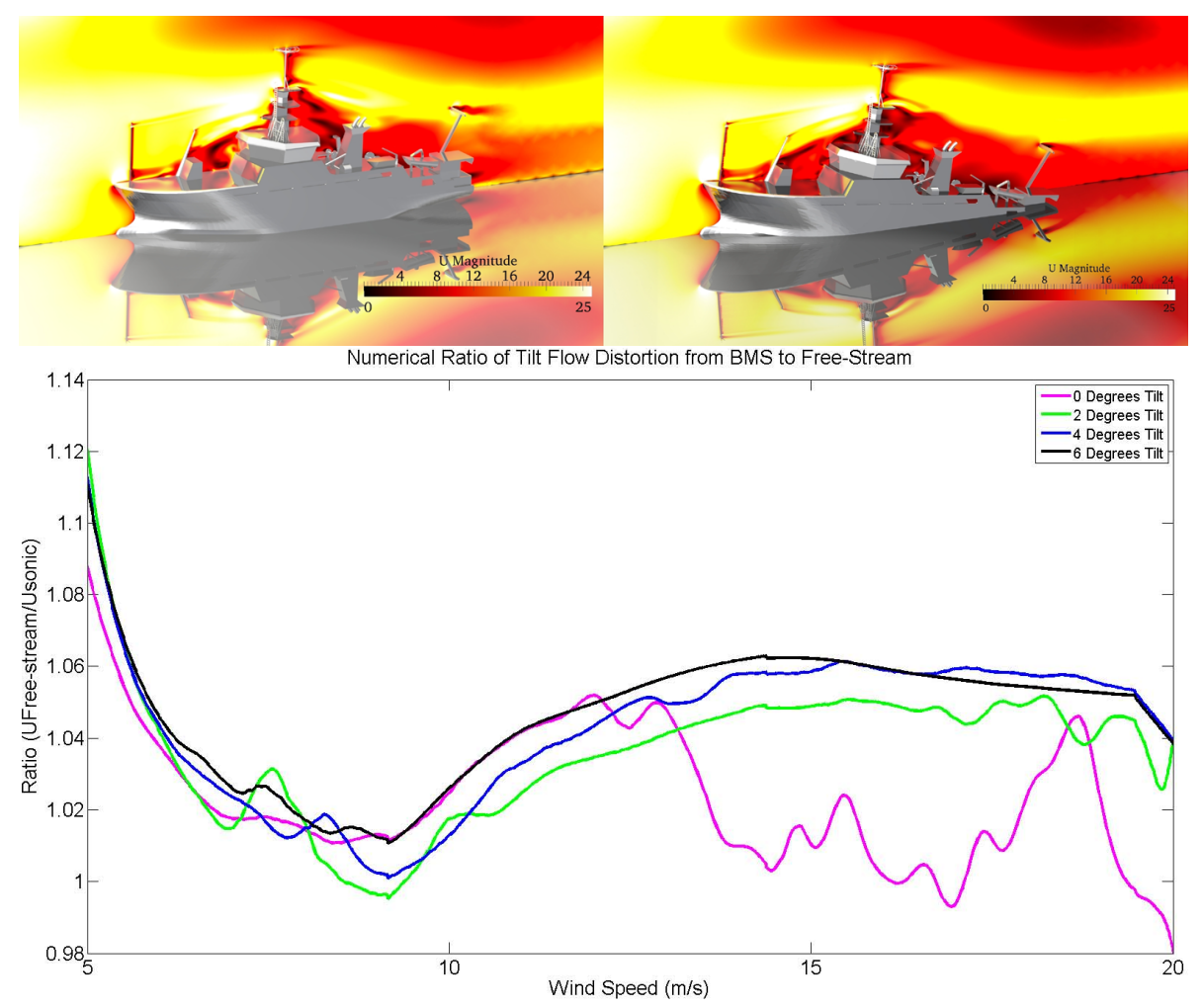

Fig. 10. (a) Flow speed over the ship for $0^{\circ}$ and $+6^{\circ}$ tilt for a steady-state input stream of $25 \mathrm{~m} \mathrm{~s}^{-1}$. (b) Modelled ratio from BMS to undisturbed flow over 5 to $25 \mathrm{~m} \mathrm{~s}^{-1}$ for tilt angles of $0^{\circ}, 2^{\circ}, 4^{\circ}$ and $6^{\circ}$.

simulations were performed for a $0-60^{\circ}$ bow-on wind. In Fig. 11 is shown velocity magnitude profiles around Mast1 and Mast2, each with a Campbell sonic anemometer and Licor LI7500 gas analyser. The wake structure from Mast2 has a greater influence on instrumentation downstream, whereas Mast1 has a more predictable gradient of turbulence in the wake of the design, which allows for a simpler correction. However, Mast1 design has a higher vertical turbulent gradient due to the lack of open sections which would allow for wind to pass through the design.

A cross-sectional vector plot of the airflow divergence for the $x$ direction uniform inflow velocity is shown in Fig. 12. This was scaled by the measured vertical component of the inflow velocity. Highly distorted wind magnitudes can be seen in the vertical direction of the plot. Anemometer measurement locations positioned directly above the control boxes can be highly affected due to the recirculation caused by the instrumentation. The Campbell sonic anemometer and Licor gas analyser setup position ahead of this vertical vector field is nearly in free stream, defining that positions ahead of this field have little effect from the instrumentation boxes. Comparison of mast geometries was also conducted to allow for a future comparison with the effects of the new measurement system deployed on the RV Celtic Explorer when sufficient experimental data are obtained. The developed differences in the mast geometries can then be applied to the
RV Celtic Explorer simulations containing the Mast1 design. This was conducted due to the complex geometry of the Mast2 design, which would have been computationally expensive in comparison to the Mast1 design and also due to the limited amount of experimental data currently available.

\section{Conclusions}

It has been established that the OpenFOAM simpleFOAM algorithm shows close agreement with experimental wind speed measurements and can be used as a valid correction for flow distortion over oceanographic platforms. It has shown a close prediction of flow distortion errors to within $12 \%$ of experimental results and predicts the same scale of errors across each space tested. This correlates with the statement of Gagnon and Richard (2010) that the simpleFOAM algorithm had a $10 \%$ mean ratio to experimental testing.

The ratio method shows a defined mean method for correction of wind speed data for full cruise periods. This gave percentage accuracy to bin-averaged experimental results of $6.61 \%$ for $20.5 \mathrm{~m}$ prediction space and $6.25 \%$ across a $0.5 \mathrm{~m}$ prediction space. It has been shown that matching the mean ratio from experimental to numerical results led to a prediction within $11 \%$ for the $20.5 \mathrm{~m}$ space from the SBD to BMS anemometer, and $50 \%$ for the $0.5 \mathrm{~m}$ space between the BMS 


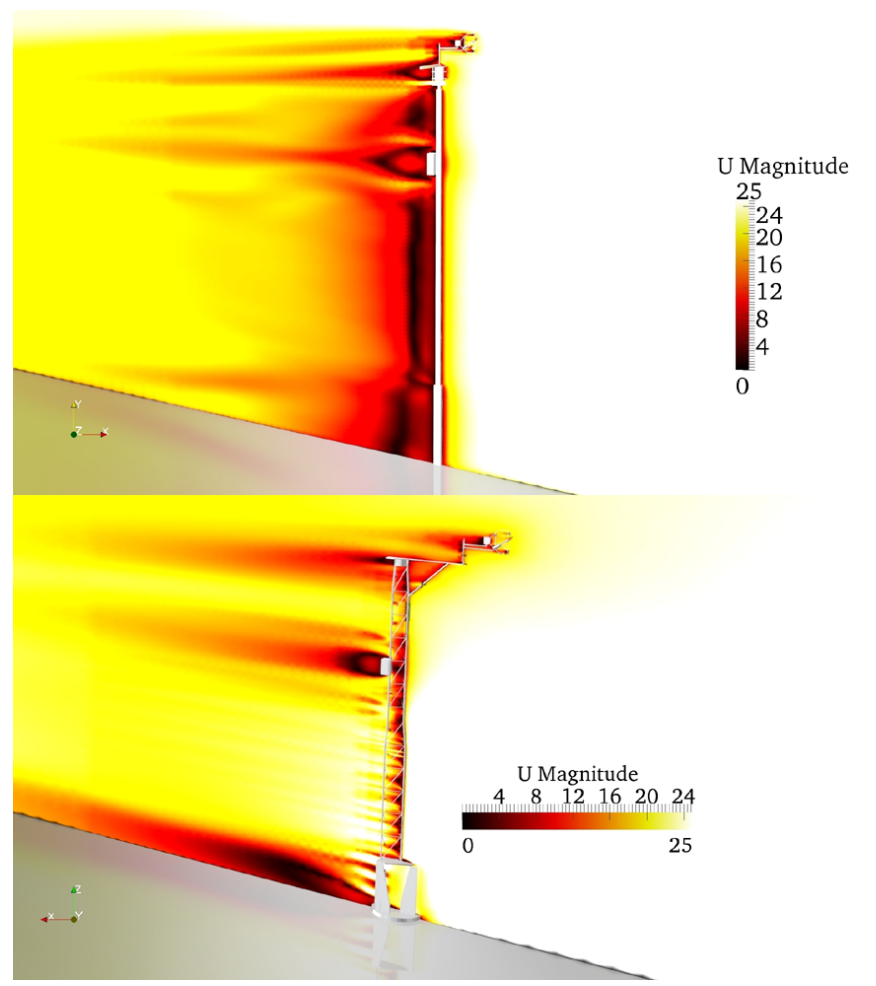

Fig. 11. Modelled velocities for each of the two masts for an input velocity of $25 \mathrm{~m} \mathrm{~s}^{-1}$

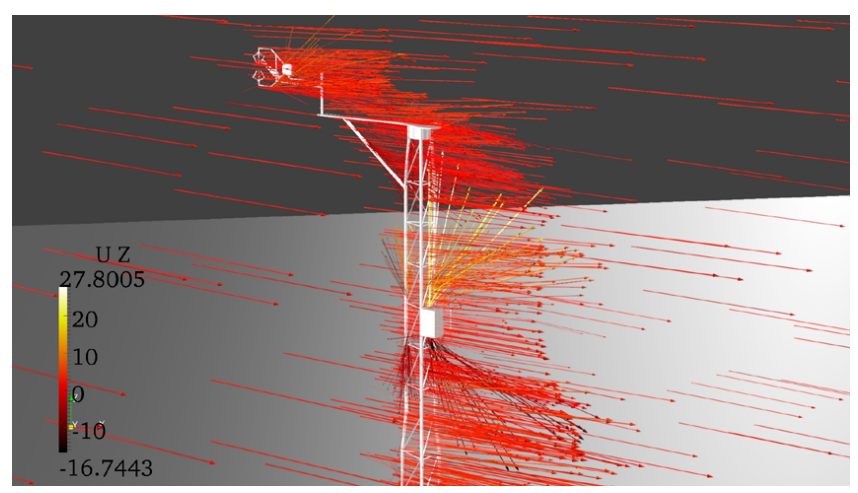

Fig. 12. Cross-sectional velocity vectors scaled from 5 to $25 \mathrm{~m} \mathrm{~s}^{-1}$ and $0^{\circ}$.

and YM1. Therefore, over a small space, the prediction of error using an averaged steady-state model is not appropriate, and a sub-scale time-dependant LES model should be used to improve accuracy. This defines also that for the eddy correlation calculations, a more accurate LES model should be used to reduce the errors in correcting the wind speed measurements used in these calculations.

It has also been found that for a highly accurate correction model, a discrete selection of inflow directions should be simulated. Higher scatter has been shown to develop in certain orientations as a direct effect of a crane being mounted on one side of the bow deck. In high-wind-speed situations, the crane causes recirculation and higher errors, which is apparent in both numerical and experimental results.

Previous research (e.g., Popinet et al., 2004; Yelland et al., 2002) has correctly concluded that wind direction is the dominant factor in flow distortion errors for micrometeorological measurements on research vessels. Our results show that the magnitude of the wind speed is also a quantity of importance, which shows a deviation of flow distortion error scaling linearly by up to $5.6 \%$ at wind speeds above $5 \mathrm{~m} \mathrm{~s}^{-1}$. It can also be concluded that wind speed dependence, shown both numerically and experimentally, increases as the wind speed increases above $5 \mathrm{~m} \mathrm{~s}^{-1}$. We have also found that the flow distortion does not scale linearly with increasing wind speed.

The results of the mast simulations indicate that the effects of a more complex geometry give more distortion effects and errors in wind speed estimates. These simulations also show a consistency that is apparent in all the performed simulations. High wind speeds tend to have lesser distortion effects. The two simulated mast geometries showed that a more applicable design would be a telegraph pole design with as little blockage as possible to the inflow wind stream. In the future, an optimised design will be developed for deployment and testing.

The comparison between different instrumentation setups showed the 3-D Campbell sonic anemometer to have less distortion effects than the 3-D Gill sonic anemometer. This is due primarily to its sampling probe window being ahead of the device, thus causing less impact on the mean airflow. With additional instrumentation positioned beside the anemometer, e.g. a Licor LI-7500 gas analyser, it was found that the best position for sampling is in the wake of the anemometer. This will allow for measurements at the anemometer location to be unaffected by the instrument's blockage. An evaluation of the Licor LI-7500 dry box also suggested that anemometers positioned directly above dry boxes can be highly affected. This is due to the recirculation, and our results suggest that they need to be positioned more than $1.5 \mathrm{~m}$ above the dry boxes for unaffected airflow.

The ideal location for the BMS anemometer was found to be as far as possible from the superstructure of the vessel and outside of its wake. It was found that the ideal location of the BMS anemometer for the RV Celtic Explorer was $3 \mathrm{~m}$ ahead of the bow and at an elevation $14 \mathrm{~m}$ above the bow deck in order to reduce the distortion effects to $4 \%$.

Study of the effect of various ship pitch angles was performed, which to our knowledge was the first time that such a study had been carried out using CFD. The vertical displacement was carried out over a limited number of angles, but the results show that vertical orientation of the vessel is a quantity that affects the passing flow. From the simulations, it can be seen that the vertical displacement of the vessel for all wind speeds changes flow distortion by $2.5 \%$ as the angle increases. The pitch of the vessel through higher angles of attack develops a higher error in the vertical component of 
the wind speed at low inflow velocities, thus causing higher lift and higher distortion.

Acknowledgements. This research is supported by the Irish Research Council for Science, Engineering and Technology via the Enterprise Partnership Scheme Postgraduate Scholarship Programme, grant number EPSPG/2011/249, and the EU FP7 project CARBOCHANGE under grant agreement no. 264879. S. Landwehr was supported by Science Foundation Ireland under grant 08/US/I1455.

Edited by: A. Sterl

\section{References}

Blender: 3D modeling software, version 2.60, 2011.

Brut, A., Butet, A., Planton, S., Durand, P., and Caniaux, G.: Influence of the airflow distortion on air-sea flux measurements aboard research vessels: Results of physical simulations applied to the EQUALANT99 experiment, J. Amer. Meteor. Soc., 15, 147-150, 2002.

Castro, I. P. and Robins, A. G.: the flow around a surface mounted cube in uniform and turbulent streams, J. Fluid. Mech., 79, 307335, 1977.

Edson, J. B., Hinton, A., Prada, K. E., Hare, J. E., and Fairall, C. W.: Direct covariance flux estimates from mobile platforms at sea, J. Atmos. Ocean. Technol., 15, 547-562, 1998.

Gagnon, L. and Richard, J. M.: Parallel CFD of a prototype car with OpenFOAM, Tech. rep., Dept of Mech. Eng., Laval University, Québec, Canada, 2010.

Griessbaum, F., Moat, B. I., Narita, Y., Yelland, M. J., Klemm, O., and Uematsu, M.: Uncertainties in wind speed dependent $\mathrm{CO}_{2}$ transfer velocities due to airflow distortion at anemometer sites on ships, J. Atmos. Chem. Phys., 10, 5123-5133, 2010, http://www.atmos-chem-phys.net/10/5123/2010/.

Kahma, K. K. and Lepparanta, M.: On errors in wind speed observations on R/V Aranda, J. GeoPhysica, 17, 155-165, 1981.

Menter, F. R.: Zonal Two Equation k-Turbulence Models for Aerodynamic Flows, J. American Institute of Aeronautics and Astronautics, 93, 1993.
Oost, W. A., Fairall, C., Edson, J., Smith, S., Anderson, R., Wills, J., Katsaros, K., and DeCosmo, J.: Flow distortion calculations and their application in HEXMAX, J. Atmos. Ocean. Technol., 11, 366-386, 1994.

OpenCFD: OpenFOAM flow solver, version 2.0.1, 2012.

OpenFOAM: OpenFOAM Users Guide 2009, version 1.6, http: //foam.sourceforge.net/doc/Guides-a4/UserGuide.pdf/,(last access: 12 September 2012), 2011.

openfoamwiki: The Simple algorithm in OpenFoam, http:// openfoamwiki.net/index.php/, (last access: 21 December 2012), 2010.

Popinet, S.: Gerris: A tree-based adaptive solver for the incompressible Euler equations in complex geometries, J. Comput. Phys., 190, 572-600, 2003.

Popinet, S.: The Gerris Flow Solver, version 1.2.0, 2008.

Popinet, S., Smith, M., and Stevens, C.: Experimental and numerical study of the turbulence characteristics of airflow around a research vessel, J. Atmos. Ocean. Technol., 21, 1575-1589, 2004.

Souza, A.: How to understand Computational Fluid Dynamics Jargon, http://www.nafems.org/downloads/edocs/how_ to_understand_cfd_jargon-nafems.pdf, (last access: 8 October 2012) 2005 .

Surry, D., Edey, R. T., and Murley, I. S.: Speed and direction correction factors for shipborne anemometers, Tech. rep., University of Western On-tario, London, ON, Canada, 1989.

Thiebaux, M. L.: Wind tunnel experiments to determine cor-rection functions for shipborne anemometers, Tech. rep., Beford Institute of Oceanographyo, Dartmouth, NS, Canada, 1990.

Yelland, M., Moat, B., Taylor, P., Pascal, R., Hutchings, J., and Cornell, V.: Wind stress measurements from the open ocean corrected for airflow distortion by the ship, J. Oceanogr., 28, 15111526, 1998.

Yelland, M., Moat, B., Taylor, P., Pascal, R., and Berry, D.: CFD model estimates of the airflow distortion over research ships and the impact on momentum flux measurements, J. Atmos. Ocean. Technol., 19, 1477-1499, 2002. 